\title{
Accuracy of a Dedicated Bone-Supported Surgical Template for Dental Implant Placement with Direct Visual Control
}

\author{
Yi Sun, Msc, PhD ${ }^{1}$; Heinz-Theo Luebbers, MD, DMD²; Jimoh Olubanwo \\ Agbaje, DMD, MSc, $\mathrm{PhD}^{1}$; Liang Kong, DDS, $\mathrm{PhD}^{3}$; Serge Schepers, MD, DDS ${ }^{4}$; \\ Luc Daems, MD, DDS ${ }^{5}$; Paul Legrand, MD, DDS ${ }^{6}$; Reinhilde Jacobs, DDS, \\ $\mathrm{PhD}^{1}$; Constantinus Politis, MD, DDS, MHA, MM, PhD ${ }^{1}$; Luc Vrielinck, \\ MD, DDS $^{4 *}$ \\ ${ }^{1}$ OMFS IMPATH research group, Department of Imaging \& Pathology, Faculty of \\ Medicine, University of Leuven and Oral \& Maxillofacial Surgery, University \\ Hospitals Leuven, Leuven, Belgium \\ ${ }^{2}$ Surgical Planning Laboratory, Brigham and Women's Hospital, Boston, MA, USA \\ ${ }^{3}$ Department of Oral and Maxillofacial Plastic Surgery, School of Stomatology, \\ Fourth Military Medical University, Xi'an, PR China \\ ${ }^{4}$ Department of Oral and Maxillofacial Surgery, Ziekenhuis Oost-Limburg, Campus \\ St. Jan, Genk, Belgium \\ ${ }^{5}$ Department of Oral and Maxilllofacial Surgery, ZNA Middelheim, Antwerp, Belgium \\ ${ }^{6}$ Department of Oral and Maxilllofacial Surgery, Mariaziekenhuis Noord Limburg, \\ Belgium
}

Submitted June 2015. Accepted for publication September 2015.

\begin{abstract}
The aim of this study is to evaluate feasibility and accuracy of dental implant placement utilizing a dedicated bone-supported surgical template. Thirty-eight implants (sixteen in maxilla, twentytwo in mandible) were placed in seven fully edentulous jaws (three maxillae, four mandibles) guided by the designed bone-supported surgical template. A voxel-based registration technique was applied to match pre- and post-operative CBCT scans. $\mathrm{T}$ he mean angular deviation and mean linear deviation at the implant hex and apex were $6.4 \pm 3.7^{\circ}\left(0.7^{\circ}-14.8^{\circ}\right), 1.47 \pm 0.64$ $\mathrm{mm}(0.5-2.56 \mathrm{~mm})$ and $1.70 \pm 1.01 \mathrm{~mm}(0.71-4.39 \mathrm{~mm})$, respectively. The presented bonesupported surgical template showed acceptable accuracy for clinical use. In return for reduced accuracy, clinicians gain accessibility when using this type of surgical template for both the maxilla and the mandible. This is particularly important in patients with reduced mouth opening.
\end{abstract}

Keywords: computer-assisted, accuracy, 3D printing, cone beam CT, dental implant, bonesupported surgical template

\footnotetext{
*Corresponding author: Luc Vrielinck, Department of Oral and Maxillofacial Surgery, Ziekenhuis Oost-Limburg, Campus St. Jan, Schiepse Bos 6, 3600 Genk, Belgium. Phone: +32 893261 61. Fax: +32 89321779 . E-mail: luc.vrielinck@zol.be. Other authors:yi.sun@uzleuven.be; tluebbers@bwh.harvard.edu; jimoh.agbaje@uzleuven.be; kongliang@fmmu.edu.cn; serge.schepers@zol.be; luc.daems@skynet.be; paul.legrand@telenet.be; reinhilde.jacobs@uzleuven.be; constantinus.politis@uzleuven.be.
} 


\section{INTRODUCTION}

In the early 1990s, computer-assisted surgical planning concepts were developed for dental implant placement [1]. Initially, this was done using a preoperative multidetector computerized tomography (MDCT) scan of the patient. The procedure then evolved into using virtual planning of implant placement via three-dimensional surface rendering to obtain the desired location by adjusting the length and angulation of the implants, while achieving an optimal functional and esthetic outcome. Eventually, a surgical template was fabricated using rapid prototyping to transfer the surgical simulation to the patient. Overall, three separate types of surgical templates can be distinguished:

1. A teeth-supported template in partial edentulism.

2. A bone-supported template in full edentulism.

3. A mucosa-supported template in full edentulism for minimal invasive surgery.

In general, previous studies have shown quite accurate results using surgical templates for dental implant placement [2-6]. When using a surgical template, each drill guide typically requires a minimum of approximately $35 \mathrm{~mm}$ of open space to allow the surgeon to smoothly place the drill into the guidance hole. This measure is based on the following reasoning: $20-\mathrm{mm}$ drill length plus $10 \mathrm{~mm}$ for the distance between the top of the implant and the top of the guided metal sleeve, plus $5-\mathrm{mm}$ thickness of the drill piece head. In subjects with a limited mouth opening, such template-based surgery remains very difficult. In an attempt to overcome this clinical downside, this study presents the development and accuracy assessment of a bonesupported surgical template with a dedicated lateral window.

\section{MATERIALS AND METHODS}

\subsection{Subjects}

This study was conducted according to the World Medical Association Declaration of Helsinki on medical research protocols. All patients agreed and signed informed consent forms before participating. Between October 2008 and December 2009, six patients (two females, four males) with either a fully edentulous maxilla and/or mandible were enrolled in this study. They all had sufficient bone volume for primary implant surgery. No preoperative bone augmentation was required. The mean age of the patients was $61.7 \pm 10.5$ years old ( $47-79$ years). All subjects were treated by one oral and maxillofacial surgeon in the Department of Oral and Maxillofacial Surgery, Ziekenhuis Oost-Limburg, Campus St. Jan, Genk, Belgium.

\subsection{Methods}

\subsubsection{Preoperative CBCT Scanning}

All patients underwent cone beam computed tomography (CBCT) scanning (Galileos, Sirona Dental Systems GmbH, Bensheim, Germany) without dentures in place. Scanning parameters were as follows: $85 \mathrm{KV}, 42 \mathrm{mAs}$, and VO1 high contrast mode. Each slice comprised $512 \times 512$ pixels, with a voxel size of $0.3 \times 0.3 \times 0.3 \mathrm{~mm}$. The output data were exported in DICOM format. 


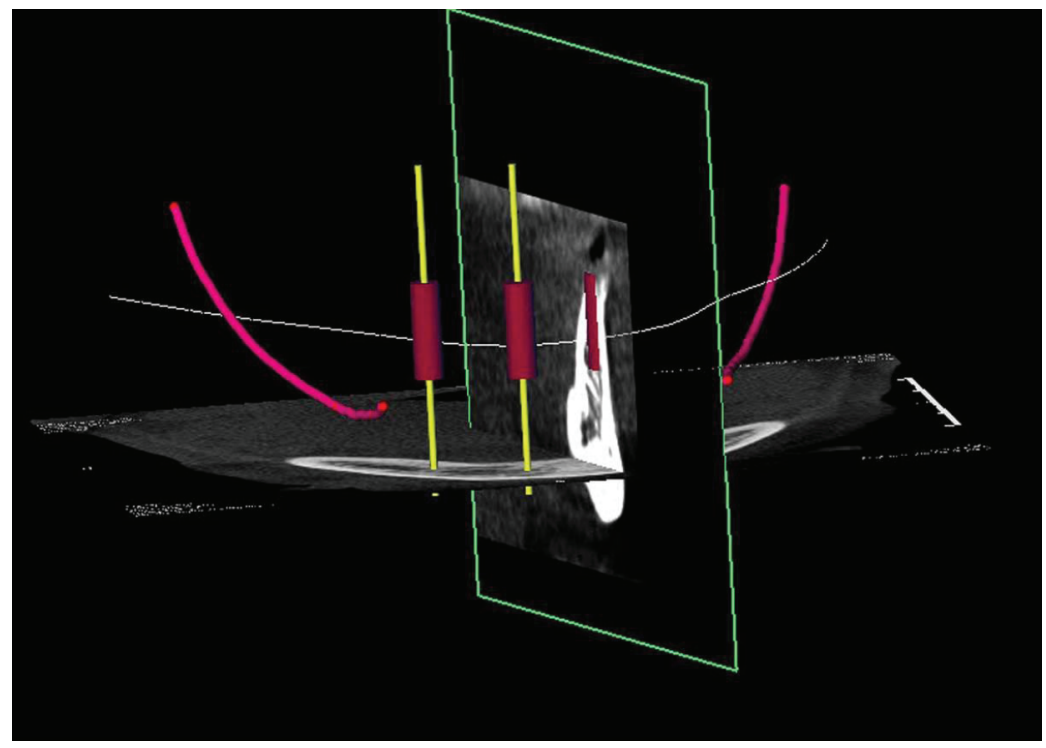

Figure 1. Implants (red parallel tubes) are virtually placed in the mandible. The mandibular nerve is indicated by the pink curves. Diameter and length for each implant are adjustable to achieve optimized function.

\subsubsection{Planning Procedure}

A customized dental implant planning platform was programmed in MevisLab (MeVis Medical Solutions AG, Bremen, Germany). The DICOM data of the preoperative CBCT scan were imported into this software. An experienced surgeon individually selected the proper threshold value to segment the maxilla and/or mandible in a threedimensional (3D) view. For each treated jaw, four to six implants were virtually placed in the optimized location (Figure 1). Both the diameter and the length were adjusted for each implant. Three anchor pins were planned to stabilize the surgical template during the operation. The implants and the segmented jaws were exported in the STL format for further processing.

\subsubsection{Design and Fabrication of the Surgical Template}

The exported implants and the segmented jaws were imported to the CAD tool, VisCAM Mesh 5.0 (Marcam Engineering GmbH, Bremen, Germany). A bonesupported surgical template was designed with a lateral opening at the buccal side of each guidance hole (Figure 2). The lateral opening allowed the drill to be easily placed into the guidance hole. The segmented jaw and the designed surgical template were printed via an Alaris 3D printer (Objet Ltd, Rehovot Israel). The layer thickness of this printed model was $0.03 \mathrm{~mm}$. 


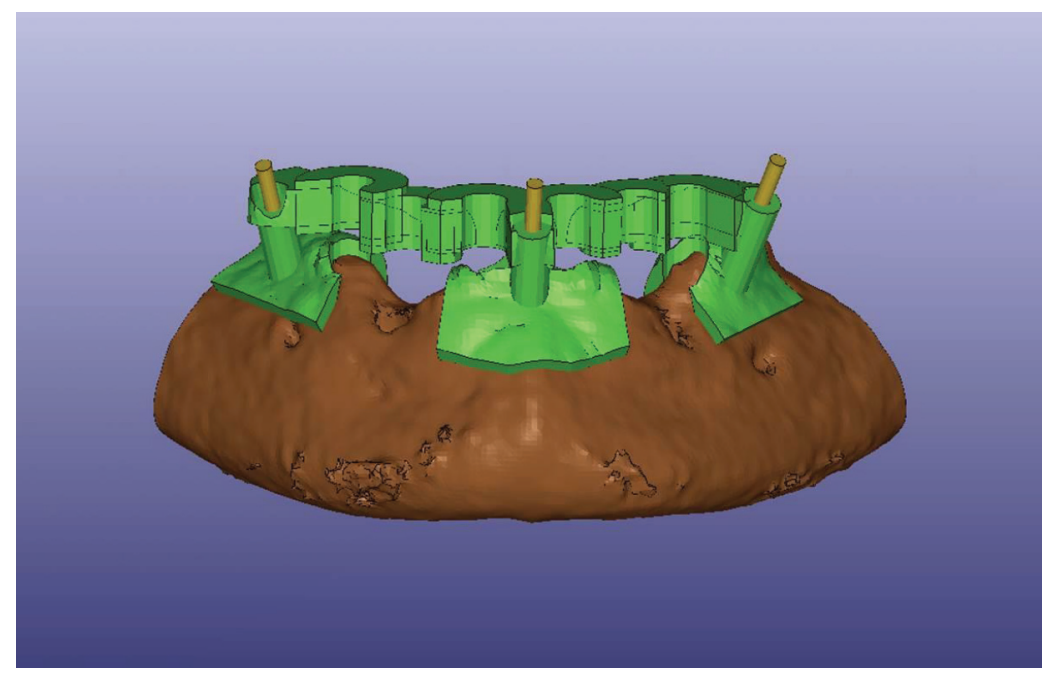

Figure 2. CAD model of bone-supported surgical template (green) designed and positioned on the mandible (brown). Three anchor pins (yellow) are planned to fix template on the mandible.

\subsubsection{Surgical Procedure}

All patients were treated under local anesthesia. A muco-periosteal flap was designed and raised to expose the prospective implant sites and the bony base of the surgical template. Once the surgical template was positioned, a drill with a diameter of $1.5 \mathrm{~mm}$ was used to position the anchor pins, fixing the surgical template to the exposed bony surface.

The surgeon defined the implant's entry point by estimating the center of the halfopening guidance hole. The surgeon also visually controlled the drilling direction, which was parallel to the direction of the guidance hole (Figure 3). The drilling was performed using sequential drills with increasing diameters, as provided by the Brånemark System ${ }^{\circledR}$ protocol (Nobel Biocare, Kloten, Switzerland). Using the indications on the drill, the surgeon was able to control the drilling depth as well. After drilling, the surgical template was removed and the implants were placed (Figure 4). Implant stability was checked and cover screws were placed. The wound site was rinsed and the muco-periosteal flap was repositioned and closed over the cover screw.

\subsubsection{Postoperative CBCT Scan}

To confirm an acceptable position needing no correction, the patients received a postoperative CBCT scan (Galileos, Sirona Dental Systems GmbH, Bensheim, Germany) immediately after surgery. The scanning parameters were as follows: $85 \mathrm{KV}, 42 \mathrm{mAs}$, and VO1 high contrast mode. Voxel-based registration using maximization of mutual information [7] was performed to register the postoperative 


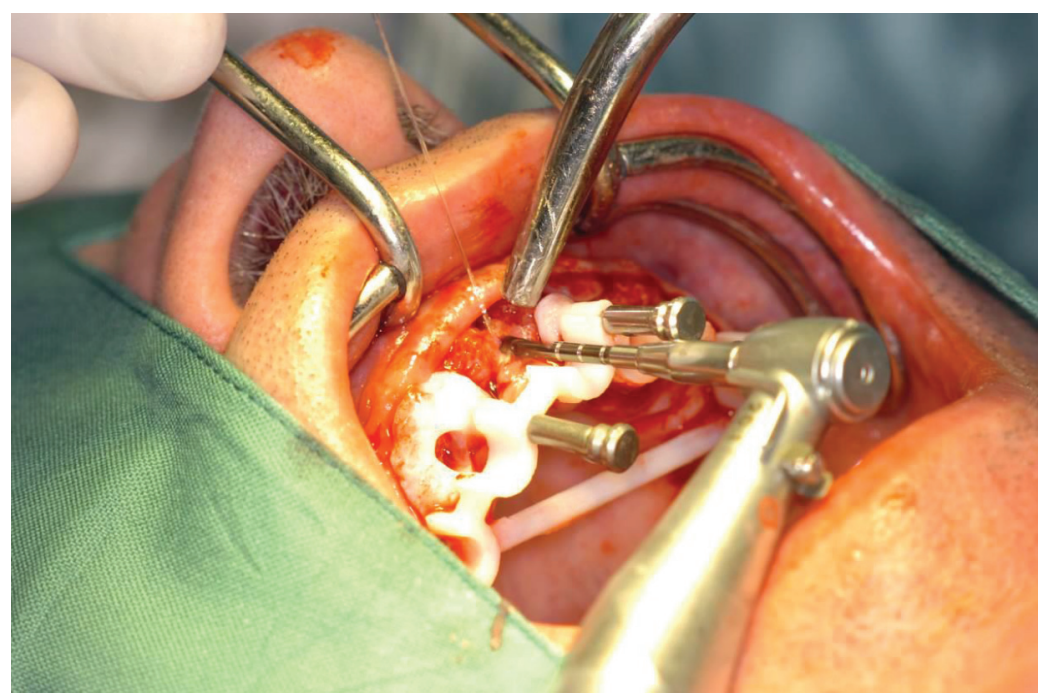

Figure 3. During operation, the anchor pins fixes the printed surgical template on the patients' maxilla. The drilling is performed using sequential drills with increasing diameters. The surgeon visually controlled drilling direction, which is parallel to the direction of the half-opening guidance hole.

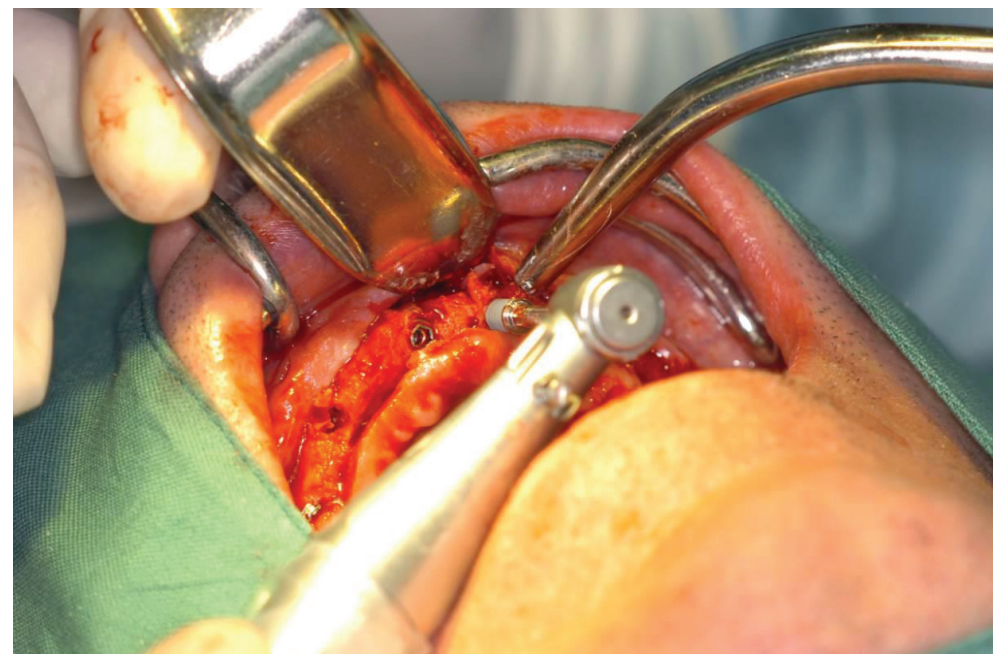

Figure 4. After final drill, the implants are placed without further guidance. 


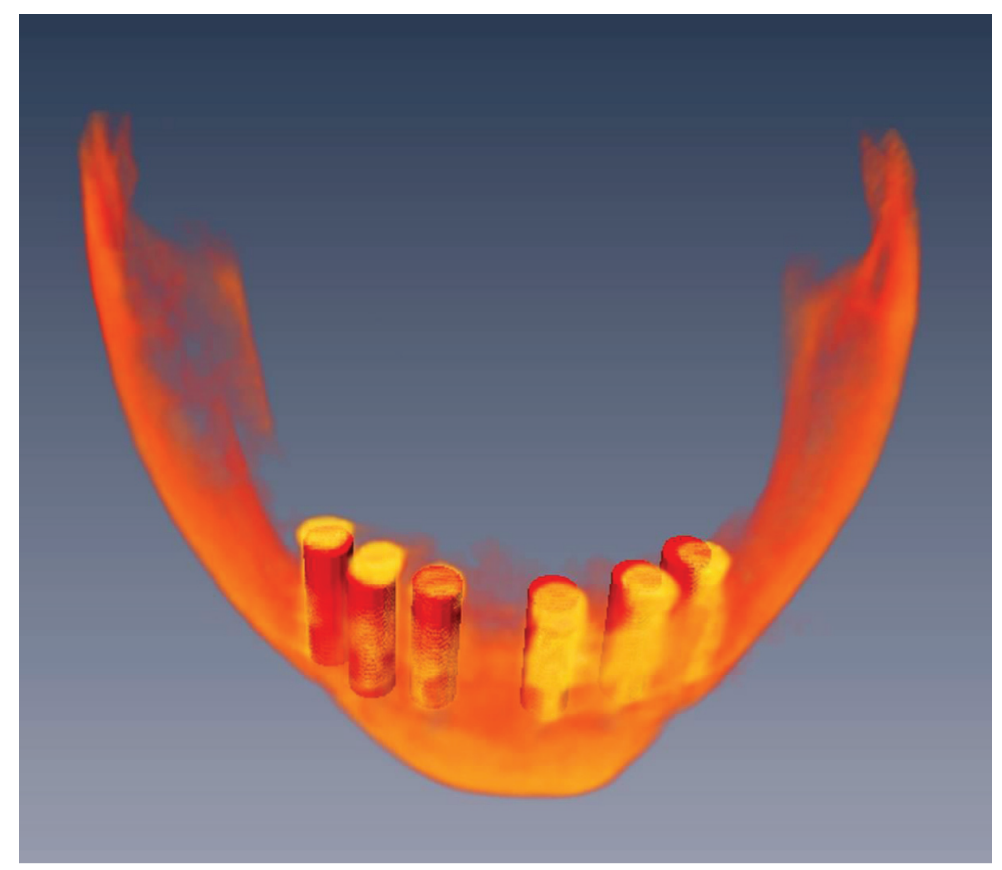

Figure 5. The placed implants are matched to their preoperative surgical planning to evaluate the achieved accuracy.

CBCT data to the preoperative $\mathrm{CBCT}$ data. The registered postoperative $\mathrm{CBCT}$ data were imported into the customized implant planning platform in DICOM format. The preoperative surgical planning was loaded to evaluate the accuracy of the dental implant placement (Figure 5).

\subsubsection{Accuracy Analysis}

The detailed measurement was performed using the following protocol:

To account for metal artifacts from the placed implants on the postoperative twodimensional cross section image, the hex center and the apex center of the implant were indicated and additionally controlled on the axial, sagittal, and coronal images. Afterwards, the axis of the implant was created (Figure 6). The linear and angular deviations at the implant hex and apex were measured within the customized implantplanning platform (Figure 7).

To avoid intraobserver bias, measurements were performed as a consensus process by a medical technician and an experienced surgeon, both of whom did not perform the surgical procedure. 


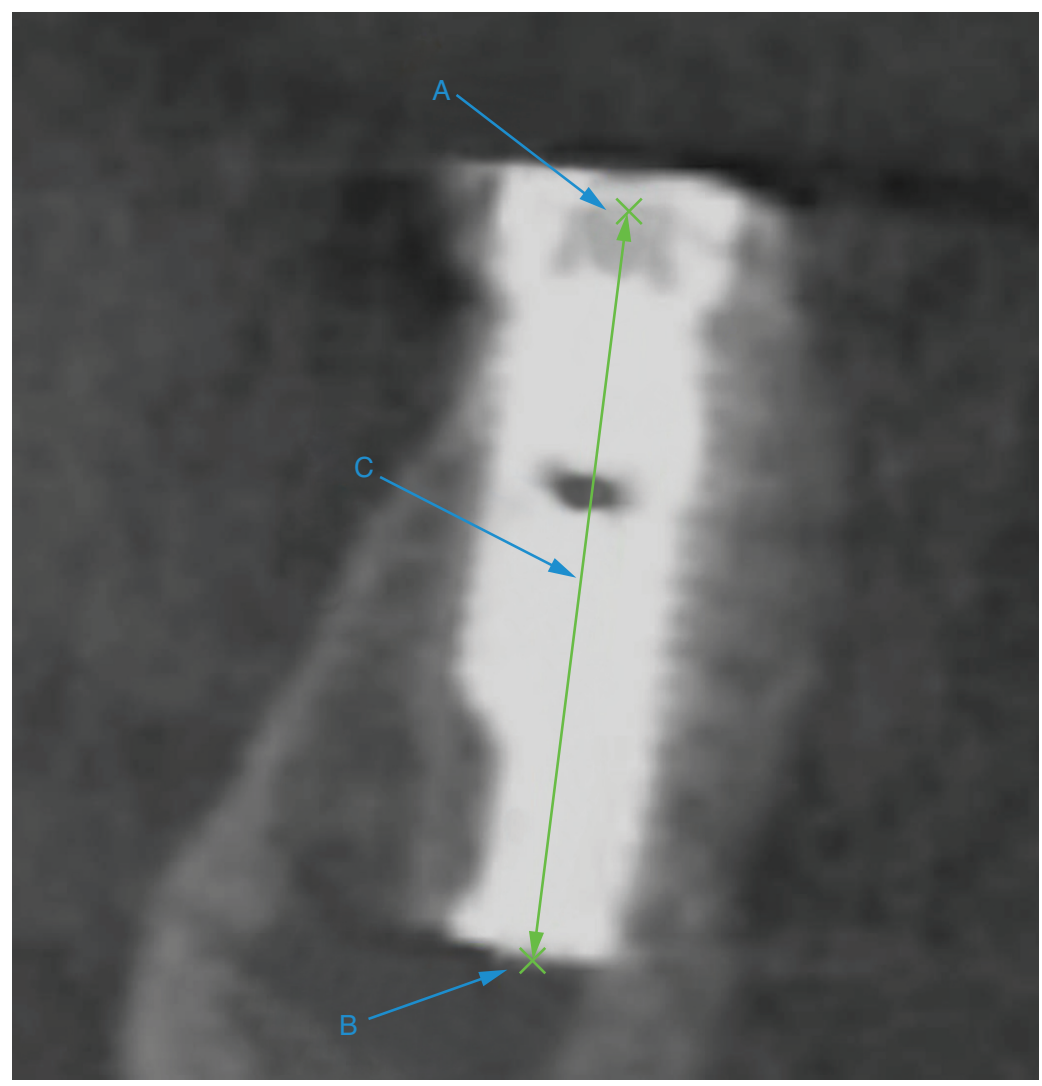

Figure 6. Point A is the hex center of the placed implant at the level of the implant neck. Point $\mathrm{B}$ is the apex center of the placed implant. $\mathrm{C}$ is the axis of the placed implant defined by Points A and B.

\section{RESULTS}

\subsection{Feasibility of Using the Present Bone-Supported Surgical Template}

A total of 38 implants (Branemark System ${ }^{\circledR}$ MkIII Groovy RP, length: $13 \mathrm{~mm}$, diameter: $3.75 \mathrm{~mm}$ or $4 \mathrm{~mm}$, Nobel Biocare, Kloten, Switzerland) were placed in seven fully edentulous jaws (maxilla: 3, mandible: 4). Sixteen implants were placed in maxillae and 22 implants were placed in mandibles. All surgeries were without incident. With the lateral opening, the surgeon felt comfortable in positioning the drill into the guidance hole.

\subsection{Accuracy of the Dental Implant Placement Utilizing the Present Bone-} Supported Surgical Template

Compared to the planned implant positions, the mean angular deviation of all placed implants was $6.4 \pm 3.7^{\circ}\left(0.7^{\circ}-14.8^{\circ}\right)$, whereas the mean linear deviation was 


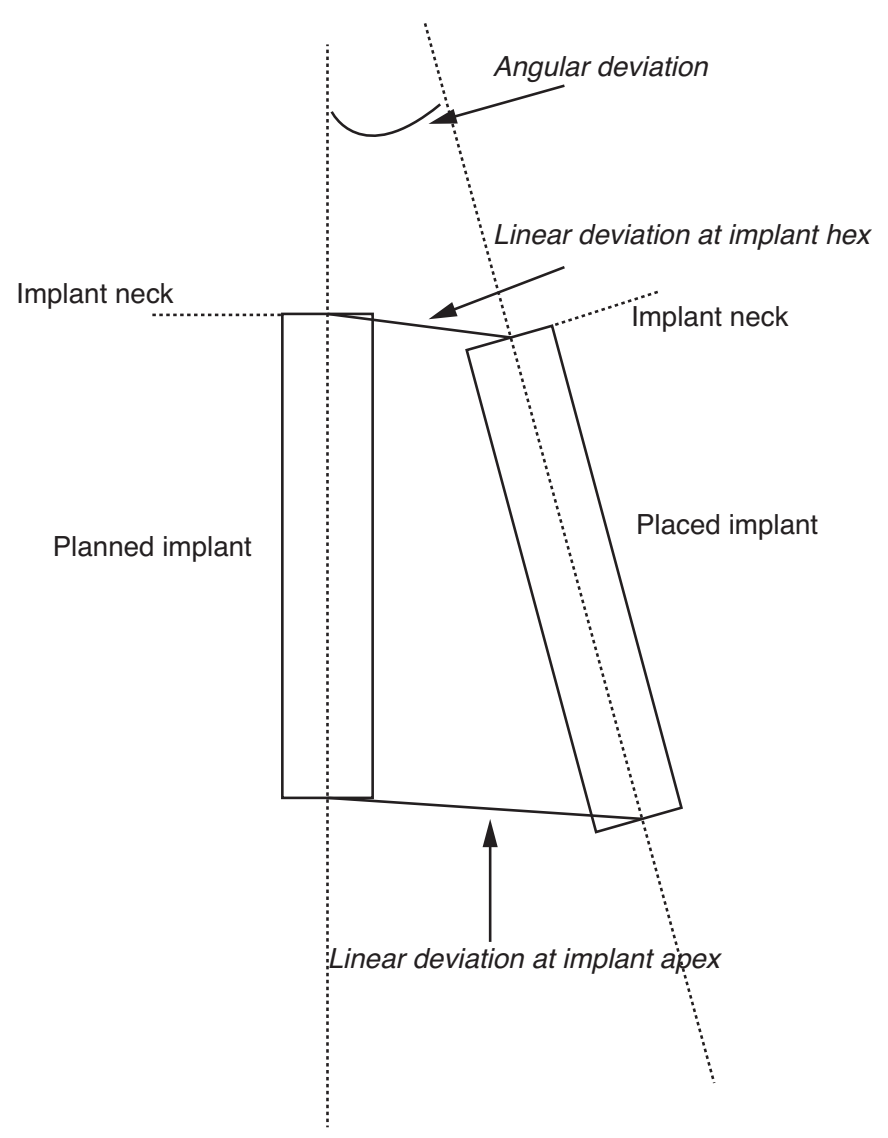

Figure 7. Between the planned and placed implant position, the angular deviation and linear deviation at the implant hex (at the level of the implant neck) and apex are evaluated separately.

$1.47 \pm 0.64 \mathrm{~mm}(0.5-2.56 \mathrm{~mm})$ at the implant hex and $1.70 \pm 1.01 \mathrm{~mm}(0.71-4.39$ $\mathrm{mm})$ at the implant apex.

\section{DISCUSSION}

For full edentulous patients, previous studies have presented fairly accurate results to place dental implants using a mucosa-supported template [8-11]. For patients with a limited mouth opening, traditional template-based surgery remains difficult to place the drill into the guidance hole. In order to overcome this clinical downside, a lateral window at the buccal side of each guidance hole is designed to allow the surgeon to place the drill into the guidance hole more easily. However, because of the lateral window, the dental implant placement can no longer be fully guided. A muco-periosteal flap is necessary to increase the surgeons' visibility and allow direct vision control during implant 
placement. In our study, we present the development and accuracy assessment of a bonesupported surgical template with a dedicated lateral window. Compared to studies using a mucosa-supported guide $[10,11]$, the accuracy of dental implant placement using the presented surgical template is less, but still clinically acceptable. Clinicians should also be aware that, due to the flap surgery, the patient's healing period could be prolonged.

Previous in vivo studies have reported the accuracy of dental implant placement utilizing a regular bone-supported surgical template. In most of the studies, no differentiation between the maxilla and mandible were made. The mean linear and angular deviations in our study are slightly larger compared to those in the literature of closed drill guides $(0.71-1.3 \mathrm{~mm}$ mean linear deviation at the implant hex, $0.77-1.6 \mathrm{~mm}$ mean linear deviation at the implant apex, $2.39^{\circ}-5.1^{\circ}$ mean angular deviation) [5, 12-16]. In our study, the maximum angular deviation and maximum linear deviation at the implant hex and apex were $14.8^{\circ}, 2.56 \mathrm{~mm}$ and $4.39 \mathrm{~mm}$, respectively. These results are larger than those from Arison et. al. $\left(6.9^{\circ}, 2.1 \mathrm{~mm}\right.$, $2.6 \mathrm{~mm}$ ) [13]. However, the maximum deviation observed in our study is similar to that of Cassetta et al. (14.34 $, 3 \mathrm{~mm}, 3.98 \mathrm{~mm})$ [12] and Pettersson et al. (11.74, $2.68 \mathrm{~mm}$, $3.62 \mathrm{~mm})$ [17].

A previous study [18] reported that the accuracy of voxel-based registration was half of the voxel size of the scan when utilizing an i-CAT CBCT scanner (Imaging Sciences International, Hatfield, Pennsylvania, USA). In our study, the Galileos CBCT scanner (Sirona Dental Systems GmbH, Bensheim, Germany) was used. Liang and colleagues [19] have shown that there is no significant difference in noise level between the image quality of the Galileos and the i-CAT CBCT scanner. Furthermore, it has been proven that noise has no significant influence on the voxel-based registration criterion [7]. Thus, the accuracy of the voxel-based registration method used in the present study was estimated to be around $0.15 \mathrm{~mm}$.

During our study, there were several factors that influenced the accuracy of the dental implant placement. First, the entry point and the drilling direction were not as precisely controlled by the template; they could have been accidentally or purposely manipulated by the surgeon. This human error contributes to the deviation between the placed and planned dental implants. To eliminate this error, repeatedly practicing our designed bone-supported template on models is recommended before actual use.

Second, geometric distortion occurred during fabrication of the surgical template. To ensure a smooth, accurate, and highly detailed representation of the designed CAD model of the surgical template, the layer thickness of this printed surgical template was $0.03 \mathrm{~mm}$. From this point of view, the error caused by the geometric distortion during fabrication of surgical template can be ignored.

The third and most effective factor is the data segmentation. In our study, the CAD design of the bone-supported template is based on the segmented maxilla or mandible. Correct segmentation of the maxilla or mandible is essential for positioning the surgical template accurately during the operation. Stumpel $[20,21]$ reported that an incorrect selection of the threshold value for the segmentation of the scan denture was found to be a factor in the deformation of SLA-produced surgical templates. In our study, a CBCT scanner is used instead of an MDCT scanner, which was utilized in previous 
studies [12-16, 22]. In our study, the imaging technology of the Galileos CBCT is based on an image intensifier. A previous study [23] has proved that the accuracy of a 3Dsurface reconstruction from a Galileos scanner is equivalent to other CBCT scanners using a flat panel detector, but somewhat lower compared to MDCT. Compared to the MDCT scans, the image quality of the CBCT scans have a higher noise level and poorer contrast $[19,24,25]$. These factors cause difficulty in segmenting the maxilla or mandible when utilizing the CBCT data. If particularly high accuracy is needed, MDCT could be considered for the preoperative scan, providing a better image quality. There is question as to whether the higher dose of MDCT is justified.

In the presented pilot study, a limited number of jaws were involved. A larger scale of patients should be included in the future study to evaluate the performance of the presented design. The same concept should be validated on a teeth-supported surgical template to evaluate the accuracy of dental implant placement.

\section{CONCLUSIONS}

The presented bone-supported surgical template with a dedicated lateral window showed acceptable accuracy for clinical use. In return for an increased accessibility, clinicians should expect a reduced level of accuracy when using this type of surgical template in both the maxilla and mandible. This is particularly important in patients with a reduced mouth opening.

\section{CONFLICT OF INTEREST}

The authors indicated no potential conflicts of interest.

\section{REFERENCES}

[1] Verstreken K, Van Cleynenbreugel J, Marchal G, Naert I, Suetens P, van Steenberghe D. Computerassisted planning of oral implant surgery: a three-dimensional approach. The International journal of oral \& maxillofacial implants, 1996, 11:806-810.

[2] Van Steenberghe D, Malevez C, Van Cleynenbreugel J, Bou Serhal C, Dhoore E, Schutyser F, Suetens P, Jacobs R. Accuracy of drilling guides for transfer from three-dimensional CT-based planning to placement of zygoma implants in human cadavers. Clinical oral implants research, 2003, 14:131-136.

[3] Schneider D, Marquardt P, Zwahlen M, Jung RE. A systematic review on the accuracy and the clinical outcome of computer-guided template-based implant dentistry. Clinical oral implants research, 2009, 20:73-86.

[4] D'Haese J, Van De Velde T, Komiyama A, Hultin M, De Bruyn H. Accuracy and complications using computer-designed stereolithographic surgical guides for oral rehabilitation by means of dental implants: a review of the literature. Clinical implant dentistry and related research, 2012, 14:321-335.

[5] Van Assche N, Vercruyssen M, Coucke W, Teughels W, Jacobs R, Quirynen M. Accuracy of computeraided implant placement. Clinical oral implants research, 2012, 23 Suppl 6:112-123.

[6] Chen X, Yuan J, Wang C, Huang Y, Kang L. Modular preoperative planning software for computeraided oral implantology and the application of a novel stereolithographic template: a pilot study. Clinical implant dentistry and related research, 2010, 12:181-193.

[7] Maes F, Collignon A, Vandermeulen D, Marchal G, Suetens P. Multimodality image registration by maximization of mutual information. IEEE transactions on medical imaging, 1997, 16:187-198.

[8] Sun Y, Luebbers HT, Agbaje JO, Schepers S, Politis C, Van Slycke S, Vrielinck L. Accuracy of Dental Implant Placement Using CBCT-Derived Mucosa-Supported Stereolithographic Template. Clinical implant dentistry and related research, 2015, 17:862-870. 
[9] Cassetta M, Di Mambro A, Di Giorgio G, Stefanelli LV, Barbato E. The Influence of the Tolerance between Mechanical Components on the Accuracy of Implants Inserted with a Stereolithographic Surgical Guide: A Retrospective Clinical Study. Clinical implant dentistry and related research, 2015, 17:580-588.

[10] D'Haese J, Van De Velde T, Elaut L, De Bruyn H. A prospective study on the accuracy of mucosally supported stereolithographic surgical guides in fully edentulous maxillae. Clinical implant dentistry and related research, 2012, 14:293-303.

[11] Van de Wiele G, Teughels W, Vercruyssen M, Coucke W, Temmerman A, Quirynen M. The accuracy of guided surgery via mucosa-supported stereolithographic surgical templates in the hands of surgeons with little experience. Clinical oral implants research, 2014, Doi: 10.1111/clr.12494.

[12] Cassetta M, Giansanti M, Di Mambro A, Calasso S, Barbato E. Accuracy of Two Stereolithographic Surgical Templates: A Retrospective Study. Clinical implant dentistry and related research, 2013, 15:448-459.

[13] Arisan V, Karabuda ZC, Ozdemir T. Accuracy of two stereolithographic guide systems for computeraided implant placement: a computed tomography-based clinical comparative study. Journal of periodontology, 2010, 81:43-51.

[14] Ersoy AE, Turkyilmaz I, Ozan O, McGlumphy EA. Reliability of implant placement with stereolithographic surgical guides generated from computed tomography: clinical data from 94 implants. Journal of periodontology, 2008, 79:1339-1345.

[15] Ozan O, Turkyilmaz I, Ersoy AE, McGlumphy EA, Rosenstiel SF. Clinical accuracy of 3 different types of computed tomography-derived stereolithographic surgical guides in implant placement. Journal of oral and maxillofacial surgery, 2009, 67:394-401.

[16] Stubinger S, Buitrago-Tellez C, Cantelmi G. Deviations between placed and planned implant positions: an accuracy pilot study of skeletally supported stereolithographic surgical templates. Clinical implant dentistry and related research, 2014, 16:540-551.

[17] Pettersson A, Komiyama A, Hultin M, Nasstrom K, Klinge B. Accuracy of virtually planned and template guided implant surgery on edentate patients. Clinical implant dentistry and related research, 2012, 14:527-537.

[18] Nada RM, Maal TJ, Breuning KH, Berge SJ, Mostafa YA, Kuijpers-Jagtman AM. Accuracy and reproducibility of voxel based superimposition of cone beam computed tomography models on the anterior cranial base and the zygomatic arches. PloS one, 2011, 6:e16520.

[19] Liang X, Jacobs R, Hassan B, Li L, Pauwels R, Corpas L, Souza PC, Martens W, Shahbazian M, Alonso A, Lambrichts I. A comparative evaluation of Cone Beam Computed Tomography (CBCT) and Multi-Slice CT (MSCT) Part I. On subjective image quality. European journal of radiology, 2010, 75:265-269.

[20] Stumpel LJ. Deformation of stereolithographically produced surgical guides: an observational case series report. Clinical implant dentistry and related research, 2012, 14:442-453.

[21] Stumpel LJ. Congruency of stereo lithographically produced surgical guide bases made from the same CBCT file: a pilot study. Clinical implant dentistry and related research, 2013, 15:531-537.

[22] Vrielinck L, Politis C, Schepers S, Pauwels M, Naert I. Image-based planning and clinical validation of zygoma and pterygoid implant placement in patients with severe bone atrophy using customized drill guides. Preliminary results from a prospective clinical follow-up study. International journal of oral and maxillofacial surgery, 2003, 32:7-14.

[23] Liang X, Lambrichts I, Sun Y, Denis K, Hassan B, Li L, Pauwels R, Jacobs R. A comparative evaluation of Cone Beam Computed Tomography (CBCT) and Multi-Slice CT (MSCT). Part II: On 3D model accuracy. European journal of radiology, 2010, 75:270-274.

[24] Gupta R, Grasruck M, Suess C, Bartling SH, Schmidt B, Stierstorfer K, Popescu S, Brady T, Flohr T. Ultra-high resolution flat-panel volume CT: fundamental principles, design architecture, and system characterization. European radiology, 2006, 16:1191-1205.

[25] Scarfe WC, Farman AG. What is cone-beam CT and how does it work? Dental clinics of North America, 2008, 52:707-730. 



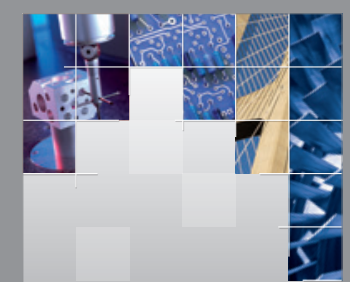

\section{Enfincering}
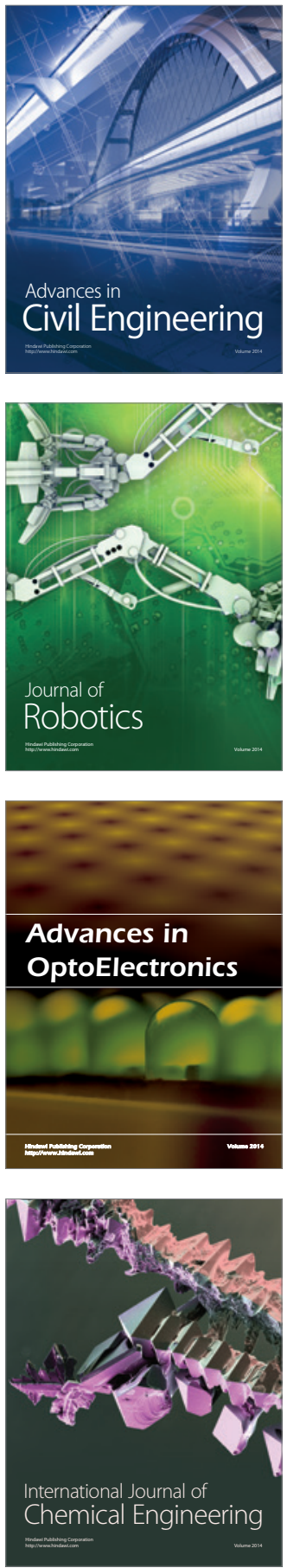

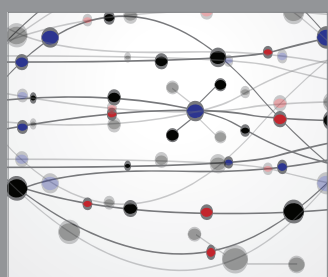

The Scientific World Journal

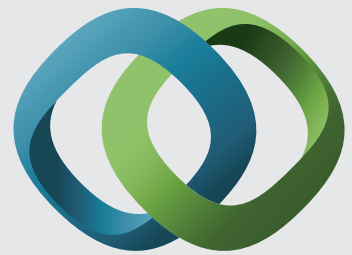

\section{Hindawi}

Submit your manuscripts at

http://www.hindawi.com
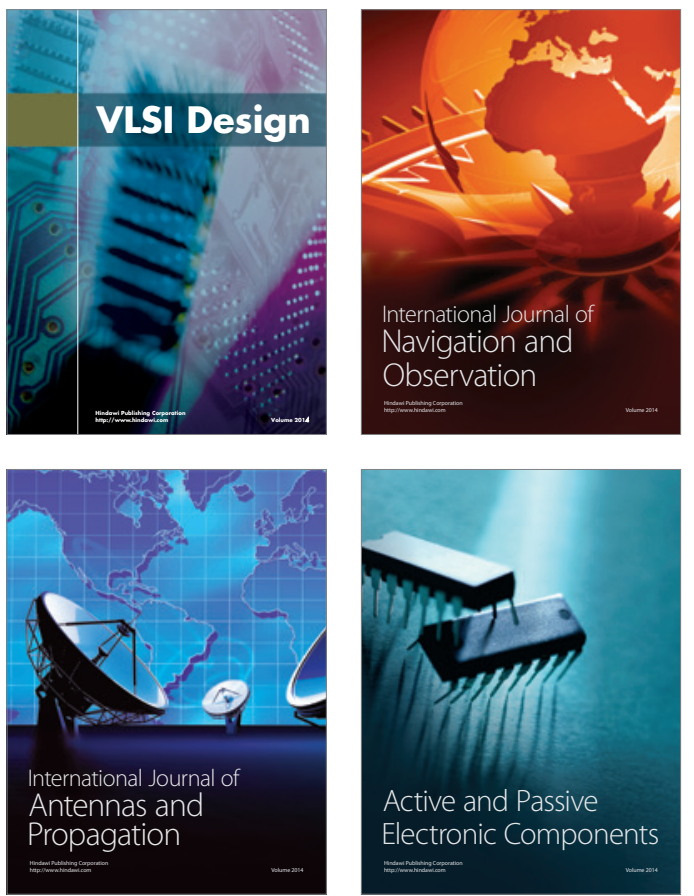
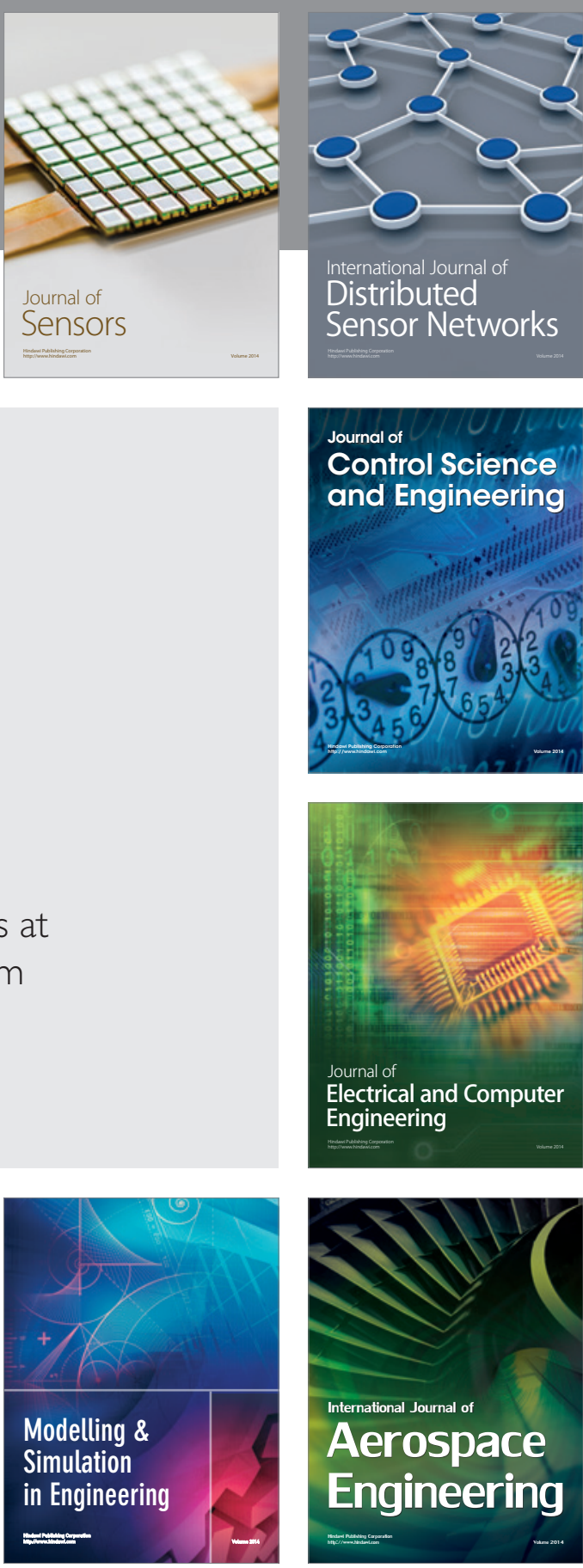

International Journal of

Distributed

Sensor Networks

Journal of

Control Science

and Engineering
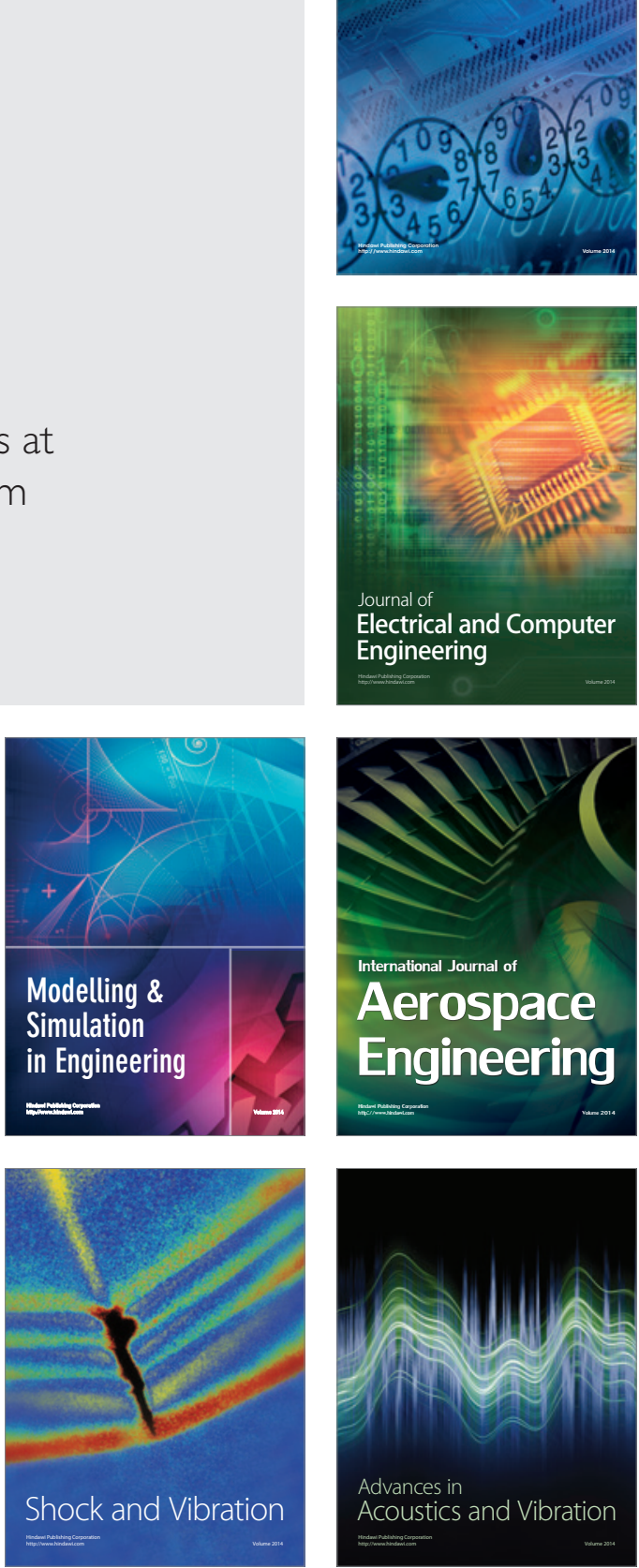